\title{
Anderson Localization for Multi-Dimensional Systems at Large Disorder or Large Energy
}

Francois Delyon, Yves Lévy, and Bernard Souillard

Centre de Physique Théorique`, Ecole Polytechnique, F-91128 Palaiseau Cedex, France

\begin{abstract}
We prove that discrete Schrödinger operators on $\mathbb{Z}^{d}$ with a randompotential have almost-surely only pure point spectrum and exponentially decaying eigenfunctions for large disorder or large energy. This is the first proof of localization for multi-dimensional Anderson models.
\end{abstract}

\section{Introduction}

Disordered systems are presently widely studied from the mathematical point of view. One of the challenging questions concerns the Anderson localization theory, which in mathematical terms amounts to studying the nature of the spectrum of random self-adjoint operators, such as a discrete Schrödinger equation with a random potential, which is among condensed matter physicists the most popular model for describing the electron propagation in a disordered system. A brief survey of these problems can be found in ref. [12], whereas [11] presents a very large bibliography on them.

In this paper we consider the multi-dimensional discrete Schrödinger operator with a random potential given for $\Psi \in \mathbb{R}^{\mathbb{Z}^{d}}, d \geqq 1$, by

$$
\left(H^{V} \Psi\right)(x)=-\sum_{|y-x|=1} \Psi(y)+V(x) \Psi(x)
$$

where $V=\{V(x)\}_{x \in \mathbb{Z}^{d}}$ is a random potential chosen with a probability distribution $\mu$ in $\Omega=\mathbb{R}^{\mathbb{Z}^{d}}$. For all potentials, $H$ is a self-adjoint operator on $l^{2}\left(\mathbb{Z}^{d}\right)$ and admits as a core the set of those $\Psi$ with a finite support.

For $d=1$, it is known that exponential localization occurs even for arbitrarily weak disorder. Several works have dealt with these one-dimensional problems; for the simplest proof of localization for one and quasi one-dimensional systems, and for a set of older references, we refer to a companion paper [3] which uses the same techniques as the present paper.

* Groupe de recherche 048 du CNRS 
For $d>1$, localization is expected to occur for all energies at sufficiently strong disorder and for any disorder at sufficiently high energies (if they are in the spectrum!). In the case $d=2$ the scaling theory predicts exponential localization for arbitrarily small disorder.

The only rigorous results for multi-dimensional systems are the following:

i) the spectrum of $H$ is exactly known for a large class of random potentials [9] (see also [7])

ii) The exponential asymptotic decay of the Green's function for a.e. value of the energy is proven by Fröhlich and Spencer in ref. [4] (see also [5]) in the case of large disorder or at large energy. The almost-sure absence of diffusion is also proven there. We will use this result on the Green's function in the present paper. iii) absence of an absolutely continuous component in the spectrum of $H$ for almost-every potential [10].

In the present paper we prove that for $d \geqq 1$, almost-surely, $H$ has only pure point spectrum of multiplicity one and exponentially decaying eigenfunctions for all energies at large disorder and at large energy for any disorder in a large class of discrete Schrödinger equations with a random potential. We use a recent very clarifying idea of Kotani [8] to obtain the first proof of Anderson localization for multi-dimensional disordered systems. The approach of the present paper is basically the following: we consider a situation in which roughly speaking the Green's function is exponentially decaying with distance for a.e. energy $E$ in some interval and a.e. potential. This is the type of results obtained in ref. [4] on the Green's functions. From this we deduce that for a.e. energy and potential, the polynomially bounded solutions of the equation $H \Psi=E \Psi$ are in fact exponentially decaying in space; it is essentially here the same idea as the one used in [10] to prove that the spectrum of $H$ has almost-surely no absolutely continuous part. We then use the fact that the spectral measure of the Hamiltonian, if averaged with respect to the random potential, becomes absolutely continuous with respect to the Lebesgue measure $[9,2]$, together with the idea of Kotani [8] mentioned above, which is implicitly contained also in [1]: he remarks that thus a property true for Lebesgue-a.e. energy $E$ and a.e. potential (namely that all polynomially bounded solutions of the equation $H \Psi=E \Psi$ are in fact exponentially decreasing with distance) can be made a property true for almost every potential and spectrally almost every $E$; thus it follows that for spectrally almost every $E$ the generalized eigenfunctions which are polynomially bounded are necessarily exponentially decaying and this in turn implies the result. This strategy is developed in the next section, whereas applications of the main result and extensions are treated in the last section.

[It is fair to mention here, as we have just learned while we were completing this paper, that other groups have reached similar conclusions:

-Ya. G. Golds'heid has announced [13] a proof of multidimensional localization, as we learned from [14]. There does not seem to be any written text concerning this work and we can compare neither his methods nor his results to ours.

- J. Fröhlich, F. Martinelli, E. Scoppola and T. Spencer, as we have learned 
at the Rencontre de Physique Statistique in Paris, also have a proof of multidimensional localization [15]. They use quite different techniques than ours, constructing more or less explicitly the configurations of potentials for which there are localized states and relying on estimates stronger than those of [4], whereas we use in fact estimates weaker than these. This method seems to adapt more straightforwardly to continuous Schrödinger equations; on the other hand, it does not yield non-degeneracy of the spectrum as our proof does, neither does it prove that the smallest Lyapunov exponent associated to the strip model does not go to zero as the width goes to infinity [3].

- B. Simon, M. Taylor and T. Wolff have announced in a letter [14] a proof of multidimensional localization. The main ingredients of their proof are the same as ours, although the processing is slightly different so that they get neither the non-degeneracy of the spectrum nor our result on the behaviour of the smallest Lyapunov exponent of the strip model.

In connection with the results of the present paper it is also interesting to mention the works of G. Jona-Lasinio, F. Martinelli and E. Scoppola [16] on hierarchical models of localization.]

\section{Main Result}

In this section we prove localization for multi-dimensional systems under some hypothesis on the behaviour of the Green's function. Applications of our results and extensions are described in the next section.

Let $H^{V}$ be given as above. In all this section we fix an interval $B$ (possibly $\mathbb{R}$ itself) of $\mathbb{R}$, and denote by $L$ the Lebesgue measure on $B$. Consider, for a finite box $\Lambda \subset \mathbb{Z}^{d}$, the restriction $H_{\Lambda}$ of $H^{V}$ to $\Lambda$, with free (Dirichlet) boundary conditions. Let $G_{\Lambda}(x, y ; V, E)$ denote the Green's function of $H_{\Lambda}$ for the energy $E$ taken between the sites $x$ and $y$. We make the following assumptions:

H1. There exist a positive constant $\alpha$ and positive-valued functions $C(\varepsilon)$ on $\mathbb{R}_{+}$and $\alpha(E)>\alpha$ on $B$ such that for $L$-a.e. $E \in B$, for any finite box $\Lambda \subset \mathbb{Z}^{d}, n \in \mathbb{Z}^{d}$, and $\varepsilon>0$ one can find $\Omega(\Lambda, n, E, \varepsilon) \subset \Omega$ with $\mu(\Omega(\Lambda, n, E, \varepsilon))>1-\varepsilon$ such that if $V \in \Omega(\Lambda, n, E, \varepsilon)$ then for all $m$ in $\Lambda$

$$
\left|G_{\Lambda}(n, m ; V, E)\right|<C(\varepsilon) \exp (-\alpha(E)|m-n|)
$$

(This hypothesis is fulfilled in the case studied in [4], where moreover bounds are given on the asymptotic behaviour of $C(\varepsilon)$, of the type $\left.C(\varepsilon) \lesssim \exp \left(1 / \varepsilon^{\beta}\right)\right)$

H2. For almost every configuration of the potential outside the "slice" $\mathbb{Z}^{d-1} \times[0,1]$ the distribution of the potentials in the slice, conditioned by the potential outside, has a bounded density with respect to the product of the Lebesgue measures.

The aim of this section is to prove the following theorem, the applications being discussed in the next section. 
Theorem 1. Let $H 1$ and $H 2$ true. Then $\mu$-a.s. the spectrum of $H^{V}$ in $B$ is pure point and non-degenerate and all the corresponding eigenvectors are exponentially decaying at infinity.

Proof: Let $V \in \Omega$ be given. Let $P_{V}$ be the resolution of the identity of $H^{V}$. For $x \in \mathbb{Z}^{d}$ we denote $\sigma_{x}$ the $\left(V\right.$-dependent) measure $\left\langle x\left|P_{V}\right| x\right\rangle$, where $|x\rangle$ is the vector associated to $x$ of the canonical basis. Let $\sigma=\sum_{i=0} \sum_{t \in \mathbb{Z}^{d-1}} 2^{-|t|} \sigma(t, i)$. For any $\Psi \in l^{2}\left(\mathbb{Z}^{d}\right),\left\langle\Psi\left|P_{V}\right| \Psi\right\rangle$ is absolutely continuous with respect to $\sigma$; in particular $H^{V}$ has pure point spectrum if and only if $\sigma$ is a point measure. By this remark, Theorem 1 is a mere consequence of the following Proposition 2.

Proposition 2. Let $x \in \mathbb{Z}^{d}$ be given. Let $H 1$ be true. Let the potential $V(x)$ be given according to a distribution with bounded density $\rho$ with respect to the Lebesgue measure (Hypothesis $\mathbf{H 2}$ ). Then $\sigma_{x}$ is pure point in $B$ and $\sigma_{x}$-a.s the eigenvalues are simple and the corresponding eigenvectors decay exponentially at infinity.

Proof. We first use the Borel-Cantelli lemma to find a property of the Green's function which is $\mu$-almost sure. Let $E$ be given in the set of full measure on $B$ on which $H 1$ holds, and $n \in \mathbb{Z}^{d}$ be fixed. Consider the sets $\Omega(\Lambda, n, E, \varepsilon)$ described in $H 1$. Then, let $\left\{\Lambda_{k}\right\}_{k \in \mathbb{N}}$ be any sequence of increasing finite boxes such that $\Lambda_{k} \uparrow \mathbb{Z}^{d}$ for $k \rightarrow \infty$, and let

$$
\Omega(\varepsilon)=\bigcap_{N \in \mathbb{N}} \bigcup_{k>N} \Omega\left(\Lambda_{k}, n, E, \varepsilon\right),
$$

which still depends on $E$ and $n$ that we keep fixed. If $V \in \Omega(\varepsilon)$, then there is a subsequence of $\Lambda_{k}$, still denoted $\Lambda_{k}$, such that:

$$
G_{\Lambda_{k}}(n, m ; V, E)<C(\varepsilon) \exp (-\alpha(E)|m-n|)
$$

for all $m$ in $\Lambda_{k}$. On the other hand, $\mu(\Omega(\varepsilon))>1-\varepsilon$; so by the Borel-Cantelli lemma, we can thus obtain:

Proposition 3. Let $H 1$ be true. Then for $\mu \times L$-a.e. $(V, E)$ and for any $n \in \mathbb{Z}^{d}$, from any sequence of finite increasing boxes $\Lambda_{k}$ containing $n$ such that $\Lambda_{k} \uparrow \mathbb{Z}^{d}$ one can extract a subsequence still denoted $\Lambda_{k}$ and a finite constant $C(V, E, n)$ such that for all $m$ in $\Lambda_{k}$ :

$$
G_{\Lambda_{k}}(n, m ; V, E)<C(V, E, n) \exp (-\alpha(E)|m-n|) .
$$

We are now going to use Proposition 3 to prove that "most" solutions of the equation $H \Psi=E \Psi$ either increase exponentially, or decrease exponentially. So let $V$ and $E$ be such that (1) holds. We consider now the equation

$$
\left(H^{V}-E\right) \Psi=a|x\rangle\langle x| \Psi
$$

where $a$ is some non-zero real number. Let some $\Psi \in \mathbb{R}^{\mathbb{Z}^{d}}$ satisfy (2) and assume that $|\Psi(n)|$ increases slower than $\exp \alpha|n|$ in the sense that

$$
\sum|\Psi(n)| \exp -\alpha|n|<\infty
$$

Let $y \in \mathbb{Z}^{d}$ be given and $\Lambda_{k}$ be an increasing sequence of finite boxes containing $x$ and 
$y$; if we write the scalar product of $(2)$ with $\langle y| G_{\Lambda_{k}}(., . ; V, E)$ we get:

$$
\begin{gathered}
\left\langle y\left|G_{\Lambda_{k}}(.,, ; V, E) \cdot\left(H_{\Lambda_{k}}-E\right)\right| \Psi\right\rangle+\left\langle y\left|G_{\Lambda_{k}}(.,, ; V, E) \cdot\left(H-H_{\Lambda_{k}}\right)\right| \Psi\right\rangle \\
=a G_{\Lambda_{k}}(y, x ; V, E) \Psi(x),
\end{gathered}
$$

and we note that the first term of the sum is equal to $\Psi(y)$. Let now $\Lambda_{k}$ be a sequence such that the conclusions of Proposition 3 are valid for $n=x$ and $n=y$; then by (3) the second term of the sum goes to zero as $k$ increases and we get:

$$
\begin{gathered}
\Psi(y)=\lim _{k \rightarrow \infty} a G_{\Lambda_{k}}(y, x ; V, E) \Psi(x), \\
|\Psi(y)|<|a \Psi(x)| C(V, E, x) \exp (-\alpha(E)|y-x|) .
\end{gathered}
$$

This proves that, for $\mu \times L$-a.e. $(V, E)$ if $\left(H^{V}-a|x\rangle\langle x|\right) \Psi=E \Psi$ and $|\Psi(n)|$ satisfies (3) then such a $\Psi$ is unique up to a multiplicative constant and

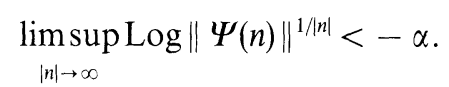

A fortiori, we obtain the

Proposition 4. Let $x \in \mathbb{Z}^{d}$ be given. Let $H 1$ be true. If $\mu_{\neq}$denotes the projection of $\mu$ on the set $\Omega_{\neq}$of the potentials outside $x$, then for $\mu_{\neq} \times L$-a.e. $\left(V_{\neq}, E\right)$ in $\Omega_{\neq} \times B$ and for any choice of $V(x)$, the solution $\Psi$ of $H^{V} \Psi=E \Psi$ such that $\sum|\Psi(n)| \exp -\alpha|n|<\infty$ is unique and satisfies:

$$
\limsup _{|n| \rightarrow \infty} \log \|\Psi(n)\|^{1 / n \mid}<-\alpha .
$$

In order to be in a position to achieve the next step we need to recall that the spectral measure averaged with respect to the potential is in fact absolutely continuous with respect to the Lebesgue measure $L[9,2]$. Since in our setting an elementary proof can be made we present it now in order to be self-contained:

Proposition 5. Let $H 2^{\prime}$ be true. Then the measure $\int_{v \in \mathbb{B}} \rho(v) \sigma_{x} d v$ is absolutely continuous with respect to $L$, where $v$ stands for $V(x)$.

Proof. Let the potential $V$ be fixed; consider a finite box $\Lambda$ and denote $P_{\Lambda}$ the resolution of the identity of the restriction $H_{\Lambda}$ of $H^{V}$ to $\Lambda$. Let $\sigma_{\Lambda}=\left\langle x\left|P_{\Lambda}\right| x\right\rangle$. For $\Lambda \uparrow \mathbb{Z}^{d}, \sigma_{\Lambda}$ converges weakly as a measure to $\sigma_{x}$. But $\sigma_{\Lambda}$ can be written explicitly:

$$
\sigma_{\Lambda}(d E)=\sum_{k} \delta\left(E-E_{k}\right)\left(\Psi_{k}(x)\right)^{2} /\left\|\Psi_{k}\right\|^{2} d E
$$

where the sum on $k$ runs on a basis of eigenvectors $\Psi_{k}$ of $H_{A}$, the corresponding eigenvalues being denoted $E_{k}$. If $\Psi_{k}(x)=0, \Psi_{k}$ remains an eigenvector for the same eigenvalue as $v=V(x)$ varies and does not contribute to $\sigma_{\Lambda}(d E)$. We can thus restrict the sum in $\sigma_{\Lambda}$ to those $k$ such that $\Psi_{k}(x) \neq 0$ (for all $v$ ). In the case when there is a degenerate eigenvalue of degeneracy $n$, we can choose an orthogonal basis of the corresponding eigenspace such that at least $n-1$ vectors take the value 0 at site $x$. Thus we can suppose in the following that no $\Psi_{k}(x)$ is 0 and that the eigenvalues $E_{k}$ are non-degenerate. Each $E_{k}$ is a monotonous function of $v$ 
and, as noted in [2], we have

$$
d E_{k} / d v=\left(\Psi_{k}(x)\right)^{2} /\left\|\Psi_{k}\right\|^{2} .
$$

Moreover, as is easily seen, the 2 sets of eigenvalues obtained respectively for $v \rightarrow-\infty$ and $v \rightarrow+\infty$ are identical except for the eigenvalue corresponding to the vector $|x\rangle$ which goes to $-\infty$ and $+\infty$ respectively. This means that when $v$ goes from $-\infty$ to $+\infty$ one and only one among the eigenvalues $E_{k}$ crosses any given value $E$. In turn this implies the existence of disjoint open sets $O_{k}$ of $\mathbb{R}$ such that the applications $v \rightarrow E_{k}(v)$ are diffeomorphisms from $\mathbb{R}$ to $O_{k}$. This allows us to make the following change of variables:

$$
\begin{aligned}
\int_{\mathbb{R}} f(E) d E \int_{\mathbb{R}} d v \sum_{k} \delta\left(E-E_{k}(v)\right)\left(\Psi_{k}(x)\right)^{2} /\left\|\Psi_{k}\right\|^{2} \\
=\int_{\mathbb{R}} f(E) d E \sum_{k} \int_{\mathbb{R}} d v \delta\left(E-E_{k}(v)\right) d E_{k} / d v \\
=\int_{\mathbb{R}} f(E) d E \sum_{k} \#\left\{v \in \mathbb{R}: E_{k}(v)=E\right\} \\
=\int_{\mathbb{R}} f(E) d E,
\end{aligned}
$$

where $f$ is any continuous function with compact support. Thus in view of the expression for $\sigma_{\Lambda}(d E)$, we get:

$$
\int_{v \in \mathbb{B}} \rho(v) d v \sigma_{\Lambda}(d E) \leqq\|\rho\|_{\infty} d E
$$

and by the weak convergence of $\sigma_{A}$ to $\sigma_{x}$ :

$$
\int_{v \in \mathbb{R}} \rho(v) d v \sigma_{x}(d E) \leqq\|\rho\|_{\infty} d E .
$$

We now use the idea emphasized by Kotani [8], namely that given $V_{\neq}$, by Proposition 5, a property true for $L$-a.e. $E$ is true for a.e. $v$ and $\sigma_{x}$-a.e. $E$ in $B$. Thus a property true for $\mu_{\neq}$-a.e. $V_{\neq}$and $L$-a.e. $E$ is true for $\mu$-a.e. $V$ and $\sigma_{x}$-a.e. $E$ in $B$. In particular, in view of Proposition 4 , for $\mu$-a.e. $V \in \Omega$ and $\sigma_{x}$-a.e. $E$ in $B$, a solution $\Psi$ of $H \Psi=E \Psi$ which is polynomially bounded necessarily decays exponentially at infinity and is the only eigenfunction for $E$.

On the other hand it follows from spectral theory that for any $V$ and $\sigma$-a.e. $E$, thus a fortiori $\sigma_{x}$-a.e. $E$, the generalized eigenfunctions for the generalized eigenvalue $E$ are polynomially bounded. Then, $\sigma_{x}$-a.s. in $B$, any generalized eigenfunction decays exponentially at infinity, thus it is in $l^{2}\left(\mathbb{Z}^{d}\right)$ and $\sigma_{x}$ is pure point and of multiplicity one in $B$. This ends the proof of Proposition 2 and thus of Theorem 1.

\section{Applications and Extensions}

1-Localization for the Multi-Dimensional Anderson Model. The Anderson model corresponds to the case where the random potential $\{V(x)\}_{x \in \mathbb{Z}^{d}}$ is a family of independent random variables with a common distribution having a density $\rho$ that we suppose bounded (in fact the original Anderson model corresponds to the case where the $V(x)$ are just equidistributed on some interval). 
Under these conditions, for which $H 2$ holds, the hypothesis $H 1$ follows from ref. [4] with $B=\mathbb{R}$ for large enough disorder (e.g. for large $\lambda$ if we multiply our potential by a coupling constant $\lambda)$ and with $B=\left(-\infty, E_{-}\right) \cup\left(E_{+},+\infty\right)$ for any disorder, $E_{-}$and $E_{+}$depending on the disorder. Thus we get localization in the intersection of the spectrum with these domains; it is known [9] that the spectrum is almost-surely exactly the set $\{E=\alpha+\beta, \alpha \in[-2,+2], \beta \in$ Support of $\rho\}$.

2-A Remark on Ergodic Random Potentials and on non Absolutely Continuous Distributions. The previous case is an example of a random potential which is ergodic with respect to the translations of $\mathbb{Z}^{d}$. For ergodic potentials, pure point spectrum and exponential decay of eigenfunctions can be derived in a slighty different way: they follow directly from Proposition 2 using translation invariance of the distribution of potentials. In fact ergodicity can be used to weaken the hypothesis $\mathrm{H}_{2}: \mathrm{H}_{2}$ can in this case be replaced by the condition that the common distribution of potential has a component absolutely continuous with respect to Lebesgue measure. Under this assumption, by restricting $V(x)$ to a subset of this component on which the density is bounded and using Proposition 2 one gets localization with positive probability, whereas it is known [9] (see also [6]) that for ergodic potentials localization holds with probability 0 or 1 . However the hypothesis $H 1$ has not yet been proven under such conditions for multidimensional systems. For one- and quasi one-dimensional systems, see [3].

3 -Weaker Conditions on the Green's Function. It is clear from the proof that we do not need for Theorem 1 as strong conditions on the Green's functions as hypothesis $H 1$; in particular we do not need exponential decay but just some property ensuring that the Green's function can be applied to any generalized eigenfunction in a convergent way. This can be useful for situations where the potential is less disordered for sites at large distances and for which the Green's function decays with a power law.

\section{References}

1. Carmona, R.: One-dimensional Schrödinger operators with random or deterministic potentials: New spectral types. J. Funct. Anal. 51, 229 (1983)

2. Delyon, F., Kunz, H., Souillard, B.: One-dimensional wave equations in disordered media. J. Phys. A16, 25 (1983)

3. Delyon, F., Lévy, Y., Souillard, B.: Anderson localization for one- and quasi one-dimensional systems. J. Stat. Phys. (to appear)

4. Fröhlich, J., Spencer, T.: Absence of diffusion in the Anderson tight-binding model for large disorder or low energy. Commun. Math. Phys. 88, 151 (1983)

5. Holden, H., Martinelli, F.: On the absence of diffusion near the bottom of the spectrum for a random Schrödinger operator on $L^{2}\left(\mathbb{P}^{v}\right)$. Commun. Math. Phys. 93, 197 (1984)

6. Kirsch, W., Martinelli, F.: On the ergodic properties of the spectrum of general random operators. J. Reine Angew. Math. 334, 141 (1982)

7. Kirsch, W., Martinelli, F.: On the spectrum of Schrödinger operators with a random potential. Commun. Math. Phys. 85, 329 (1982)

8. Kotani, S.: Lyapunov exponents and spectra for one-dimensional Schrödinger operators. Proceedings of the American Mathematical Society, meeting on "Random Matrices", Brunswick, 1984, J. Cohen Editor

9. Kunz, H., Souillard, B.: On the spectrum or random finite difference operators. Commun. Math. Phys. 78, 201 (1980) 
10. Martinelli, F., Scoppola, E.: A remark on the absence of absolutely continuous spectrum in the Anderson model for large disorder or low energy, Commun. Math. Phys. 97, 465 (1985)

11. Simon, B., Souillard, B.: Franco-American meeting on the mathematics of random and almostperiodic potential. J. Stat. Phys. 36, 273 (1984)

12. Souillard, B.: Electrons in random and almost-periodic potentials, Proceedings of "Common trends in particle and condensed matter physics" Les Houches 1983. Phys. Rep. 103, 41 (1984)

13. Golds'heid, Ya. G.: Talk at the Tachkent conference on information theory, September 1984

14. Simon, B., Taylor, M., Wolff, T.: Some rigorous results for the Anderson model, preprint Caltech

15. Fröhlich, J., Martinelli, F., Scoppola, E., Spencer, T.: Anderson localization for large disorder or low energy, preprint $n^{\circ} 426$ Università di Roma

16. Jona-Lasinio, G., Martinelli, F., Scoppola, E.: Multiple tunnelings in $d$-dimensions: A quantum particle in a hierarchical potential, preprint (1984)

Communicated by J. L. Lebowitz

Received January 9, 1985 